\title{
Long-term Tillage, Straw and N Rate Effects on Quantity and Quality of Organic $C$ and $N$ in a Gray Luvisol Soil
}

\author{
S. S. Malhi ${ }^{1}$, M. Nyborg ${ }^{2}$, T. Goddard ${ }^{3}$ and D. Puurveen ${ }^{2}$ \\ ${ }^{1}$ Agriculture and Agri-Food Canada, P.O. Box 1240, Melfort, Saskatchewan, Canada S0E 1A0; \\ ${ }^{2}$ Department of Renewable Resources, University of Alberta, Edmonton, Alberta, Canada T6G 2E3; \\ ${ }^{3}$ Alberta Agriculture, Food and Rural Development, Edmonton, Alberta, Canada T6H 5T6
}

\section{Background}

- With recent developments, such as the Kyoto protocol, there is a desire to identify best management practices to enhance carbon (C) sequestration.

- Soil organic matter is the primary source and temporary sink for plant nutrients and it maintains soil tilth, aids air and water movement, promotes water retention and reduces soil erosion.

- Soil organic carbon (SOC) reserves in western Canada have diminished considerably since the initial cultivation of native prairie grasslands 100 or more years ago, especially with tilled summer fallow.

- Adoption of better management strategies such as continuous cropping, reduced tillage, improved fertilization, application of organic amendments and crop rotations including perennial forage can increase the amount of organic $\mathrm{C}$ and/or $\mathrm{N}$ stored in the soil.

- Soil, crop and fertilizer management practices alter soil properties, but the magnitude of change depends on soil type and climatic conditions.

\section{Objective}

- To determine the long-term (19 or 27 years - 1980 to 1998 or 2006) effects of tillage, straw management and $\mathrm{N}$ fertilizer on total organic $\mathrm{C}$ (TOC) and $\mathrm{N}$ (TON), light fraction organic C (LFOC) and N (LFON), macro organic matter C (MOM-C) and N (MOM-N), microbial biomass $\mathrm{C}(\mathrm{MB}-\mathrm{C})$, and mineralizable $\mathrm{C}\left(\mathrm{C}_{\min }\right)$ and $\mathrm{N}\left(\mathrm{N}_{\min }\right)$, $\mathrm{pH}$, extractable $\mathrm{P}$, ammonium- $\mathrm{N}$ and nitrate- $\mathrm{N}$ in a Gray Luvisol (Typic Cryoboralf) loam soil.

\section{Materials and Methods}

- A field experiment conducted from 1980 to 1998 or 2006 with barley-wheat-canola rotation on a Gray Luvisol (Typic Cryoboralf) loam (pH 6.6 and initial TOC 13.75 g $\mathrm{C} \mathrm{kg}^{-1}$ ) at Breton, Alberta, Canada.

- Treatments (Table 1) included two tillages (zero tillage [ZT] and conventional tillage $[C T]$ ), two straw managements (straw removed $\left[S_{\text {Rem }}\right]$ and straw retained $\left[S_{\text {Ret }}\right]$ ) and four $\mathrm{N}$ fertilizer rates $\left(0,50\right.$ and $100 \mathrm{~kg} \mathrm{~N} \mathrm{ha}^{-1}$ in $S_{\text {Ret, }}$, and $0 \mathrm{~kg} \mathrm{~N} \mathrm{ha}^{-1}$ in $S_{\text {Rem }}$ plots). 
- Individual plots were $2.8 \mathrm{~m}$ x $6.9 \mathrm{~m}$. Plots under CT were tilled twice, once in autumn and once in spring (chisel cultivator). The ZT plots did not undergo any disturbance, except for seeding drill.

- Crop was harvested from 1980 to 2006 for seed and straw yield.

- In autumn 1998, and in spring 2007, soil samples were taken from each plot after growing wheat. The soil samples were then analyzed for various organic $\mathrm{C}$ and $\mathrm{N}$ fractions and some chemical properties.

- Total organic $\mathrm{C}$ (TOC) and $\mathrm{N}$ (TON), light fraction organic $\mathrm{C}$ (LFOC) and $\mathrm{N}$ (LFON), macro organic matter C (MOM-C) and N (MOM-N), microbial biomass $\mathrm{C}$ (MB-C), and mineralizable $\mathrm{C}\left(\mathrm{C}_{\min }\right)$ and $\mathrm{N}\left(\mathrm{N}_{\min }\right)$ measured in 0-7.5 and 7.5-15 $\mathrm{cm}$ or 0-5, 5-10 and 10-15 cm layers.

- $\quad \mathrm{pH}$, extractable $\mathrm{P}$, ammonium-N and nitrate- $\mathrm{N}$ in $0-7.5,7.5-15,15-30$ and $30-40 \mathrm{~cm}$ or $0-15,15-30,30-60,60-90$ and $90-120$ cm layers.

\section{Summary}

- Zero tillage, $\mathrm{S}_{\text {Ret }}$ and $\mathrm{N}$ fertilizer treatments usually had higher mass of TOC, TON, LFOC, LFON, $\mathrm{C}_{\min }$ and $\mathrm{N}_{\min }$ compared to the corresponding CT, $\mathrm{S}_{\text {Rem }}$ and zero-N control treatment, especially in the surface soil layers, but no consistent effect of these treatments on MB-C (Tables 2 and 3).

- MOM-C in both 0-7.5 and 7.5-15 cm layers and MOM-N in 0-7.5 cm layer generally higher with $\mathrm{S}_{\mathrm{Ret}}$ than $\mathrm{S}_{\mathrm{Rem}}$, also higher with $\mathrm{N}$ fertilizer than zero- $\mathrm{N}$, and tended to be higher under ZT than CT treatments (Table 3).

- There were close and significant correlations among most soil organic $\mathrm{C}$ or $\mathrm{N}$ fractions in most cases, except MB-C which correlated only with MOM-N at $\mathrm{P} \leq$ 0.10 , and $\mathrm{N}_{\text {min }}$ did not correlate with MOM-C (Table 4). Linear regressions between crop residue or $\mathrm{C}$ input and soil organic $\mathrm{C}$ or $\mathrm{N}$ were significant in most cases, except for $\mathrm{MB}-\mathrm{C}$, or $\mathrm{N}_{\min }(\mathrm{P} \leq 0.16)$ (Tables 5 and 6).

- In the 0-15 cm soil layer, $\mathrm{pH}$ was depressed slightly with $\mathrm{N}$ application, and extractable $\mathrm{P}$ was higher with $\mathrm{S}_{\text {Ret }}$ than $\mathrm{S}_{\mathrm{Rem}}$ and under ZT than CT, but decreased with $\mathrm{N}$ application ((Table 7).

- In this layer, nitrate-N (though quite low) increased with application of $\mathrm{N}$, but no effect of treatments on ammonium-N (Table 7). There was usually no effect of treatments on any soil parameters at depths below $15 \mathrm{~cm}$.

- Compared to the 1979, the TOC concentrations in the 0-15 cm layer indicated a loss in the $\mathrm{CTS}_{\mathrm{Rem} 0}$ treatment in 1998, and in the $\mathrm{CTS}_{\mathrm{Rem} 0} 0, \mathrm{CTS}_{\mathrm{Ret}} 0$ and $\mathrm{ZTS}_{\mathrm{Rem}} 0$ treatments in 2007 (Table 8).

\section{Conclusion}

- Elimination of tillage, straw retention and $\mathrm{N}$ application all improved organic $\mathrm{C}$ and $\mathrm{N}$ in soil, and generally differences were more pronounced for light fraction organic $\mathrm{C}$ and $\mathrm{N}$, and between the most extreme treatments $\left(\mathrm{CTS}_{\mathrm{Rem}} 0 \mathrm{vs}\right.$. $\left.\mathrm{ZTS}_{\mathrm{Ret}} 100\right)$ for each dynamic organic fraction. This will be better for the long-term sustainability of soil quality, fertility, and productivity. 


\section{Acknowledgements}

- The authors would like to thank Z. Zhang and K. Strukoff for technical help, D. Leach for preparing and Erin Cadieu for graphic design of the poster.

Table 1. Description of treatments sampled in autumn 1998 and spring 2007 at Breton, Alberta, Canada (Gray Luvisol soil, experiment established in autumn 1979)

\begin{tabular}{|c|c|c|c|c|}
\hline \multicolumn{2}{|c|}{ Treatment } & \multirow[b]{2}{*}{ Tillage system } & \multirow[b]{2}{*}{ Straw management } & \multirow{2}{*}{ Rate of $N\left(\mathrm{~kg} \mathrm{~N} \mathrm{ha}^{-1}\right)$} \\
\hline No. & ID & & & \\
\hline 1 & ZTS $_{\text {Rem } 0}$ & Zero & Straw removed & 0 \\
\hline 4 & $\mathrm{ZTS}_{\mathrm{Ret}} \mathbf{0}$ & Zero & Straw retained & 0 \\
\hline 3 & $\mathrm{ZTS}_{\mathrm{Ret}} 50$ & Zero & Straw retained & 50 \\
\hline 6 & $\mathrm{ZTS}_{\mathrm{Ret}} 100$ & Zero & Straw retained & 100 \\
\hline 2 & $\mathrm{CTS}_{\mathrm{Rem} 0}$ & Conventional & Straw removed & 0 \\
\hline 5 & $C T S_{\mathrm{Ret} 0}$ & Conventional & Straw retained & 0 \\
\hline 8 & $\mathrm{CTS}_{\mathrm{Ret}} 50$ & Conventional & Straw retained & 50 \\
\hline 7 & CTS $_{\text {Ret }} 100$ & Conventional & Straw retained & 100 \\
\hline
\end{tabular}

Table 2. Effect of long-term tillage, straw and $\mathrm{N}$ rate on mass of total organic $\mathrm{C}$ (TOC), total organic $\mathrm{N}$ (TON), light fraction organic $\mathrm{C}$ (LFOC) and light fraction organic $\mathrm{N}$ (LFON) in soil depth 0-15 cm, spring $\underline{2007}$ at Breton, Alberta, Canada (Gray Luvisol soil, experiment established in autumn 1979)

\begin{tabular}{|c|c|c|c|c|}
\hline \multirow{2}{*}{$\begin{array}{l}\text { Treatment } \\
\text { (tillage/straw/kg N ha }{ }^{-1} \text { ) }\end{array}$} & \multicolumn{2}{|c|}{$\left(\mathrm{Mg} \mathrm{ha}^{-1}\right)$} & \multicolumn{2}{|c|}{$\left(\mathrm{kg} \mathrm{C} \mathrm{ha}^{-1}\right)$} \\
\hline & TOC & TON & LFOC & LFON \\
\hline ZTS $_{\text {Rem } 0}$ & 21.95 & 2.476 & 936 & 29.6 \\
\hline $\mathrm{ZTS}_{\text {Ret } 0}$ & 25.49 & 2.799 & 977 & 31.4 \\
\hline ZTS $_{\text {Ret }} 50$ & 31.80 & 3.229 & 1456 & 49.9 \\
\hline ZTS $_{\text {Ret } 100}$ & 32.47 & 3.352 & 2335 & 92.3 \\
\hline CTS $_{\text {Rem } 0}$ & 20.11 & 2.390 & 756 & 23.6 \\
\hline $\mathrm{CTS}_{\text {Ret } 0}$ & 21.09 & 2.582 & 865 & 27.4 \\
\hline $\mathrm{CTS}_{\mathrm{Ret}} 50$ & 25.44 & 2.804 & 1508 & 49.4 \\
\hline CTS $_{\text {Ret }} 100$ & 30.57 & 3.143 & 1436 & 53.0 \\
\hline $\begin{array}{l}\operatorname{LSD}_{0.05} \\
\text { SEM }^{\mathrm{y}}\end{array}$ & $\begin{array}{l}8.03 \\
2.647^{*}\end{array}$ & $\begin{array}{l}0.578 \\
0.1906^{*}\end{array}$ & $\begin{array}{l}374 \\
123.3^{\text {*** }}\end{array}$ & $\begin{array}{l}16.3 \\
5.4^{* * *}\end{array}$ \\
\hline
\end{tabular}


Table 3. Effect of long-term tillage, straw and $\mathrm{N}$ rate on mass of mineralizable and microbial biomass $\mathrm{C}$ and $\mathrm{N}$ in soil depth 0-15 cm, autumn $\underline{1998}$ at Breton, Alberta, Canada (Gray Luvisol soil, experiment established in autumn 1979)

\begin{tabular}{|c|c|c|c|c|c|}
\hline \multirow{3}{*}{$\begin{array}{l}\text { Treatment } \\
\text { (tillage/straw/kg N ha }{ }^{-1} \text { ) }\end{array}$} & \multicolumn{2}{|c|}{ Mineralizable mass } & \multicolumn{3}{|c|}{ Microbial biomass } \\
\hline & Cmin & Nmin & MB-C & MOM-C & MOM-N \\
\hline & $\left(\mathrm{kg} \mathrm{C} \mathrm{ha}^{-1}\right)$ & $\left(\mathrm{kg} \mathrm{N} \mathrm{ha}^{-1}\right)$ & $\left(\mathrm{kg} \mathrm{C} \mathrm{ha}^{-1}\right)$ & $\left(\mathrm{kg} \mathrm{C} \mathrm{ha}^{-1}\right)$ & $\left(\mathrm{kg} \mathrm{N} \mathrm{ha}^{-1}\right)$ \\
\hline $\mathrm{ZTS}_{\mathrm{Rem}} 0$ & 303 & 25.2 & 917 & 267 & 11.1 \\
\hline $\mathrm{ZTS}_{\mathrm{Ret}} \mathrm{O}$ & 367 & 30.7 & 1063 & 464 & 18.9 \\
\hline $\mathrm{ZTS}_{\mathrm{Ret}} 50$ & 424 & 37.5 & 888 & 595 & 20.7 \\
\hline ZTS $_{\text {Ret }} 100$ & 416 & 37.6 & 1002 & 377 & 15.7 \\
\hline $\mathrm{CTS}_{\mathrm{Rem} 0}$ & 259 & 14.7 & 943 & 316 & 8.1 \\
\hline $\mathrm{CTS}_{\mathrm{Ret} 0}$ & 261 & 17.9 & 997 & 299 & 10.8 \\
\hline $\mathrm{CTS}_{\mathrm{Ret}} 50$ & 349 & 23.8 & 1089 & 591 & 23.1 \\
\hline CTS $_{\text {Ret }} 100$ & 390 & 28.4 & 1085 & 762 & 27.4 \\
\hline LSD $_{0.05}$ & 129 & 12.0 & 268 & 290 & 12.6 \\
\hline SEM $^{\mathrm{y}}$ & $42.4^{\circ}$ & $3.95^{\star *}$ & $88.4^{\mathrm{ns}}$ & $95.7^{*}$ & $4.14^{*}$ \\
\hline
\end{tabular}

Table 4. Relationships among soil organic $\mathrm{C}$ or $\mathrm{N}$ fractions (TOC, TON, LFOC, LFON, MOM-C, MOM-N, MB-C, mineralizable $\mathrm{C}\left[\mathrm{C}_{\min }\right.$ ] and mineralizable $\left.\mathrm{N}\left[\mathrm{N}_{\min }\right]\right)$ in autumn 1998 and in spring 2007at Breton, Alberta, Canada (Gray Luvisol soil, experiment established in autumn, 1979)

\begin{tabular}{|c|c|c|c|c|c|c|c|c|c|}
\hline Parameter & \multicolumn{9}{|c|}{ Correlation coefficients } \\
\hline \multicolumn{10}{|c|}{ Relationships among soil organic $\mathrm{C}$ or $\mathrm{N}$ fractions } \\
\hline$\underline{1998}$ & TOC & TON & LFOC & LFON & MOMC & MOMN & MB-C & Cmin & Nmin \\
\hline $\begin{array}{l}\text { TOC } \\
\text { TON } \\
\text { LFOC } \\
\text { LFON } \\
\text { MONAC } \\
\text { MONAN } \\
\text { MB-C } \\
\text { Cmin } \\
\text { Nmin }\end{array}$ & & $0.997^{\star \star *}$ & $\begin{array}{l}0.806^{*} \\
0.813^{*}\end{array}$ & $\begin{array}{c}0.810^{\star} \\
0.827^{\star} \\
0.986^{\star \star \star}\end{array}$ & $\begin{array}{c}0.595 \bullet \\
0.612 \bullet \\
0.934^{\star \star \star} \\
0.930^{\star \star \star}\end{array}$ & $\begin{array}{l}0.635 \bullet \\
0.661^{\bullet} \\
0.941^{\star \star \star} \\
0.965^{\star \star \star} \\
0.961^{\star \star \star}\end{array}$ & $\begin{array}{l}-0.017^{\mathrm{ns}} \\
0.049^{\mathrm{s}} \\
0.310^{\mathrm{s}} \\
0.427^{\mathrm{s}} \\
0.493^{\mathrm{s}} \\
0.590\end{array}$ & $\begin{array}{c}0.932^{\star \star \star} \\
0.940^{\star \star \star} \\
0.817^{\star} \\
0.843^{\star \star} \\
0.656^{\bullet} \\
0.736^{\star} \\
0.156^{\text {ns }}\end{array}$ & $\begin{array}{c}0.893^{\star *} \\
0.890^{\star *} \\
0.643^{\bullet} \\
0.662 \cdot \\
0.403^{\text {s }} \\
0.519^{\text {ss }} \\
-0.047^{\text {s }} \\
0.946^{\text {*** }}\end{array}$ \\
\hline$\underline{2007}$ & TOC & TON & LFOC & LFON & & & & & \\
\hline $\begin{array}{l}\text { TOC } \\
\text { TON } \\
\text { LFOC } \\
\text { LFON }\end{array}$ & & $0.990^{\star \star \star}$ & $\begin{array}{l}0.840^{\star *} \\
0.863^{\star *}\end{array}$ & $\begin{array}{c}0.835^{\star} \\
0.860^{\star \star} \\
0.994^{\star \star \star}\end{array}$ & & & & & \\
\hline
\end{tabular}


Table 5. Relationships between crop residue or crop residue C input from 1980 to 1998 or 2006 growing seasons and soil organic C or N stored in soil sampled in autumn 1998 and in spring 2007at Breton, Alberta, Canada (Gray Luvisol soil, experiment established in autumn, 1979)

\begin{tabular}{|c|c|c|c|c|c|c|c|c|c|}
\hline Parameter & \multicolumn{9}{|c|}{ Correlation coefficients } \\
\hline \multicolumn{10}{|c|}{ Relationships between crop residue or crop residue $\mathrm{C}$ inputand soil organic $\mathrm{C}$ or $\mathrm{N}$ fractions } \\
\hline 1980 to 1998 & TOC & TON & LFOC & LFON & MOM-C & $M O M-N$ & $M B-C$ & Cmin & Nmin \\
\hline $\begin{array}{l}\text { Crop residue } \\
\text { or } C \text { input }\end{array}$ & $0.813^{*}$ & $0.832^{*}$ & $0.851^{* *}$ & $0.885^{\star *}$ & $0.797^{\star}$ & $0.797^{\star}$ & $0.348^{\mathrm{ns}}$ & $0.744^{*}$ & $0.541^{0.16}$ \\
\hline 1980 to 2006 & TOC & TON & LFOC & LFON & & & & & \\
\hline $\begin{array}{l}\text { Crop residue } \\
\text { or } C \text { input }\end{array}$ & $0.820^{*}$ & $0.815^{\star}$ & $0.772^{*}$ & $0.744^{*}$ & & & & & \\
\hline
\end{tabular}

Table 6. Linear regressions for relationships between crop residue or crop residue $\mathrm{C}$ input from 1980 to 1998 or 2006 growing seasons and soil organic C or N (TOC, TON, LFOC, LFON, MOM-C, MOM-N, MB-C, mineralizable $\mathrm{C}\left[\mathrm{C}_{\min }\right]$ and mineralizable $\mathrm{N}\left[\mathrm{N}_{\min }\right]$ ) stored in soil sampled in autumn 1998 and in spring 2007at Breton, Alberta, Canada (Gray Luvisol soil, experiment established in autumn, 1979)

\begin{tabular}{|c|c|c|c|}
\hline Crop parameter $(X)$ & Soil $\mathrm{C}$ or $\mathrm{N}$ parameter $(\mathrm{Y})$ & Linear regression $(Y=a+b X)$ & $\mathrm{R}^{2}$ \\
\hline \multicolumn{4}{|l|}{1980 to 1998} \\
\hline \multirow[t]{9}{*}{ Crop residue $\mathrm{C}$ input } & TOC & $Y=22.17+0.4204 X$ & $0.6609 *$ \\
\hline & TON & $Y=2.287+0.0358 X$ & $0.6930^{*}$ \\
\hline & LFOC & $Y=385.6+30.893 X$ & $0.7244^{\star \star}$ \\
\hline & LFON & $Y=15.41+1.2000 X$ & $0.7828^{\star \star}$ \\
\hline & MOM-C & $Y=174.9+14.220 X$ & $0.6347^{\star}$ \\
\hline & MOM-N & $Y=6.128+0.5431 X$ & $0.6354^{\star}$ \\
\hline & MB-C & $Y=943.6+2.7222 X$ & $0.1210^{\mathrm{ns}}$ \\
\hline & Cmin & $Y=247.5+4.9361 X$ & $0.5539 *$ \\
\hline & Nmin & $Y=17.85+0.4570 X$ & $0.2934^{0.16}$ \\
\hline \multicolumn{4}{|l|}{1980 to 2006} \\
\hline \multirow[t]{4}{*}{ Crop residue $\mathrm{C}$ input } & TOC & $Y=17.47+0.2704 X$ & $0.6726^{\star}$ \\
\hline & TON & $Y=2.222+0.0195 X$ & $0.6648^{*}$ \\
\hline & LFOC & $Y=432.8+22.596 X$ & $0.5964^{*}$ \\
\hline & LFON & $Y=8.977+1.1128 X$ & $0.5447^{*}$ \\
\hline
\end{tabular}


Table 7 Effect of long-term tillage, straw and $\mathrm{N}$ rate on nitrate- $\mathrm{N}$, extractable $\mathrm{P}$ and $\mathrm{pH}$ in soil in spring 2007 at Breton, Alberta, Canada (Gray Luvisol soil, experiment established in autumn, 1979)

\begin{tabular}{|c|c|c|c|c|c|}
\hline \multirow{2}{*}{$\begin{array}{l}\text { Treatment }{ }^{2} \\
\text { (tillage/straw/kg N ha }{ }^{-1} \text { ) }\end{array}$} & \multicolumn{2}{|c|}{ Nitrate-N (kg N ha $\left.{ }^{-1}\right)$} & \multirow{2}{*}{$\begin{array}{c}\begin{array}{c}\text { Extractable P } \\
\left(\mathrm{kg} \mathrm{P} \mathrm{ha}^{-1}\right)\end{array} \\
0-15 \mathrm{~cm}^{2} \\
\end{array}$} & \multicolumn{2}{|c|}{$\mathrm{pH}$} \\
\hline & $0-15 \mathrm{~cm}$ & $15-30 \mathrm{~cm}$ & & $0-5 \mathrm{~cm}$ & $5-10 \mathrm{~cm}$ \\
\hline ZTS Rem0 & 2.0 & 2.0 & 35.6 & 5.94 & 5.80 \\
\hline $\mathrm{ZTS} \mathrm{Ret}_{\mathrm{Re}} \mathrm{O}$ & 2.7 & 2.5 & 44.1 & 6.08 & 5.90 \\
\hline $\mathrm{ZTS}_{\mathrm{Ret}} 50$ & 6.2 & 2.2 & 41.9 & 5.55 & 5.48 \\
\hline 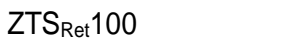 & 7.1 & 3.1 & 28.1 & 5.44 & 5.34 \\
\hline $\mathrm{CTS}_{\mathrm{Rem} 0}$ & 2.6 & 2.0 & 41.7 & 6.92 & 5.86 \\
\hline $\mathrm{CTS}_{\mathrm{Ret} 0} \mathrm{O}$ & 3.4 & 2.3 & 48.8 & 6.03 & 5.96 \\
\hline CTS Ret50 & 3.5 & 3.1 & 16.5 & 5.55 & 5.48 \\
\hline CTS Ret100 & 5.7 & 3.8 & 27.7 & 5.49 & 5.40 \\
\hline $\mathrm{LSD}_{0.05}$ & 3.5 & 1.29 & 20.7 & 0.21 & 0.28 \\
\hline SEM $^{y}$ & $1.14^{*}$ & $0.39 *$ & $6.81^{\circ}$ & $0.068^{\star \star \star}$ & $0.091^{\star \star \star}$ \\
\hline
\end{tabular}

Table 8 Total organic C (TOC) concentration in 0-15 cm soil in1979, 1990, 1998 and spring 2007 for each treatment at Breton, Alberta, Canada (Gray Luvisol soil, experiment established in autumn 1979 - mean total organic C concentration was 13.75 g C kg-1 in 1979)

\begin{tabular}{lcccc}
\hline & \multicolumn{4}{c}{ TOC concentration $\left(\mathrm{g} \mathrm{C} \mathrm{kg}^{-1}\right)$} \\
\cline { 2 - 5 } Treatment & 1979 & 1990 & 1998 & 2007 \\
\hline ZTS $_{\text {Rem } 0} 0$ & 12.59 & 12.22 & 10.51 \\
ZTS $_{\text {Ret }}$ & 13.75 & 13.92 & 13.72 & 12.18 \\
ZTS $_{\text {Ret }} 50$ & 13.75 & 17.89 & 16.76 & 15.80 \\
ZTS $_{\text {Ret }} 100$ & 13.75 & 17.39 & 16.49 & 15.22 \\
CTS $_{\text {Rem } 0}$ & 13.75 & 13.41 & 10.17 & 9.57 \\
CTS Ret $_{\text {RTS }}$ & 13.75 & 13.41 & 11.96 & 10.06 \\
CTS $_{\text {Ret }}$ CTS & 13.75 & 16.46 & 12.99 & 12.27 \\
\hline
\end{tabular}

